\title{
Problem-based learning; a new pathway towards improving patient safety-based communication skills in nursing students
}

\author{
Hossein Jamshidi ${ }^{1}$, Naser Parizad ${ }^{2}$, Masumeh Hemmati Maslakpak ${ }^{3 *}$ \\ ${ }^{1}$ Nursing and Midwifery School, Urmia University of Medical Sciences, Urmia, Iran \\ ${ }^{2}$ Patient Safety Research Center, Nursing and Midwifery Faculty, Urmia University of Medical Sciences, Urmia, Iran \\ ${ }^{3}$ Maternal and Childhood Obesity Research Center, Urmia University of Medical Sciences, Urmia, Iran
}

\section{Correspondence to:}

Masumeh Hemmati Maslakpak

Email: hemmatma@yahoo.com

hemmati_m@umsu.ac.ir

Received: 17 Oct. 2020

Accepted: 24 Nov. 2020

ePublished: 12 July 2021

Keywords: Nursing student, Patient safety, Education, Communication

\begin{abstract}
Introduction: Nursing education plays a pivotal role in patient safety in complicated healthcare environments, and problem-based learning $(\mathrm{PBL})$ is an educational method that has gained a remarkable reputation in higher education.

Objectives: The present study aimed to assess the effects of nursing education through the PBL method on patient safety-based communication skills in nursing students.

Patients and Methods: This quasi-experimental study was performed on 78 fourth-year nursing students. The participants were divided into intervention $(n=43)$ and control $(n=35)$ groups. In the intervention groups, the educational content based on the PBL method was provided in eight sessions (45-60 minutes each). Data were collected using demographic and patient safety-based communication skills questionnaires.

Results: No significant difference was observed in the mean score of patient safety-based communication skills in the control groups after the intervention $(P=0.162)$. However, the intervention groups had significantly higher scores after the educational sessions $(P=0.001)$.

Conclusion: The implementation of the PBL method affected the patient safety-based communication skills of the nursing students. Thus, it is recommended that conventional teaching methods be replaced by PBL to improve the clinical and cognitive capabilities of nursing students.
\end{abstract}

Citation: Jamshidi $\mathrm{H}$ Parizad N, Hemmati Maslakpak M. Problembased learning; a new pathway towards improving patient safetybased communication skills in nursing students. J Prev Epidemiol. 2021;6(2):e25. doi: 10.34172/jpe.2021.25

\section{Introduction}

Patient safety is a challenging issue in health care systems and plays a key role in nursing education and clinical practice (1). In addition, it is an inherent element in the quality of health care service, and an important topic in the medical community and the World Health Organization (WHO) in developed and developing countries (2). International evidence indicates that patient safety is in unfavorable conditions of the healthcare systems in many countries (3). According to the WHO report in developed countries, one per every ten patients suffers damage while receiving hospital care, which may lead to severe injuries or even death. Despite the lack of accurate statistics in developing countries, inadequate medical care has been shown to cause impairment and mortality in millions of patients in these areas (4). While no recent statistics are available on Iran's medical errors, some studies have reported the alarming rate of medical errors (5). The concept of safety skills

\begin{abstract}
Key point
Nursing education plays a pivotal role in patient safety in complicated healthcare environments. In this quasi-experimental study, 78 sample cases in two groups have been examined. Mean of patient safety-based communication skills was significantly higher in the intervention group compared to the control group $(P=0.001)$. The implementation of the problem-based learning method affected the patient safety-based communication skills of the nursing students.
\end{abstract}

(i.e., skills and behaviors that render care provision safe) has emerged in the literature on the health care system (6). Safety-related skills are classified into technical skills (e.g., systematic assessment, fluid therapy, urinary catheterization, central venous catheter insertion during resuscitation/surgery), and non-technical skills (e.g., leadership, teamwork, collaboration, situational awareness, and decision-making). Other behaviors such as conscience, alertness, humility, and communication also support

Copyright (C) 2021 The Author(s); Published by Society of Diabetic Nephropathy Prevention. This is an open-access article distributed under the terms of the Creative Commons Attribution License (http://creativecommons.org/licenses/by/4.0), which permits unrestricted use, distribution, and reproduction in any medium, provided the original work is properly cited. 
technical skills and are essential to patient safety $(7,8)$. Non-technical skills are the cognitive and interpersonal skills that complement clinical knowledge and facilitate the effective delivery of safe care (9). Extensive investigation suggests that the improvement of patient safety requires the enhancement of non-technical skills and promoting knowledge and technical skills (10). This is mainly because human factors in the workplace affect all the elements that are involved in patient care (9). Brasaite et al claimed that limited studies focused on the effects of knowledge, attitude, and professional healthcare skills on patient safety (11). The majority of nursing students have emphasized the necessity of education regarding patient safety, as well as achieving the required skills and allocating time to these educational courses (12). No challenge in the improvement of patient safety is as alarming as improving the educational preparation of nursing students. While this issue is emphasized in all healthcare professions, it is considered to be even more essential in nursing care (13). Nursing education providers play an important role in developing the knowledge, attitudes, and skills required by graduates to ensure the proper provision of patient care in a safe environment (14). In problem-based learning $(\mathrm{PBL})$, the instructor, an expert in both the educational content and teaching style, initiates the education process and encourages the learners to participate actively in the learning process (15). PBL is a novel meta-cognitive strategy in education that could improve students' decisionmaking skills (16). As a result, the PBL technique enhances students' learning, activity, interest, and cooperation in the learning process (17). Healthcare providers play a crucial role in patient safety management, and their role is rooted in their attitude, knowledge, and skills regarding patient safety (18).

Moreover, PBL positively influences autonomous and continuous learning with an emphasis on the recognition of the concepts, innovation, and acquisition of social skills. Nowadays, considering the current conditions in nursing education and the need to create the proper dynamics for innovation and the nursing promotion, nursing education program has been oriented toward active learning (14).

\section{Objectives}

The present study aimed to assess the effects of nursing education through the PBL method on patient safetybased communication skills in nursing students.

\section{Patients and Methods}

\section{Study design}

This quasi-experimental study was conducted in Urmia school of nursing and midwifery in 2019. The sample population included all the fourth-year nursing students who met the inclusion criteria: willingness to participate in the study, being in the fourth year of nursing program, and not using similar educational programs/classes. The exclusion criteria were: being absent in more than two sessions of the intervention and using similar educational programs/classes. Based on the previous study result by Hemmati et al, the mean and standard deviation of the $\mathrm{PBl}$ score was $6 \pm 2.14$ and $7.76 \pm 2.18$ in the control and intervention groups, respectively. By considering $\alpha=0.05$, power of $90 \%$ and a confidence interval of $95 \%$, the sample size was calculated 32 for each group. (17). Data were collected using demographic and patient safety-based communication skills questionnaires, which has been developed by Hemmati et al and consists of 16 items, which are scored based on a five-point Likert scale (Never; 1, Seldom; 2, Sometimes; 3, Usually; 4, Always; 5). Hemmati et al used the index of content validity (CVI) and the content validity ratio (CVR) to confirm the instrument's qualitative and quantitative content validity. The reliability of the questionnaire was confirmed at the Cronbach's alpha of 95.0 (19). After the researcher introduced himself to the participants and explained the research objectives, the students signed the written consent prior to the study. The researcher explained demographic and patient safety-based communication skills questionnaires and answered the students' concerns and questions. Then, all the students completed the questionnaires. All nursing students were divided into nine groups based on the nursing internship group schedules planned by the nursing manager. Then, students were randomly allocated to five intervention groups and four control groups. In the random allocation, the names of the groups were written and placed in envelopes. The first five envelopes were drawn from the basket were considered as the intervention groups. In total, 78 nursing students in the seventh and eighth semesters were entered into the study. The control groups included 35 students, and the intervention groups included 43 students. This study's method was as follows; the control group received no education (training session) during the study period. However, the intervention group received eight training sessions of 45-60 minutes (one session a week). In each session, a written scenario on patient safety-based communication skills were provided for the students, and they were given a week to review the scenario. In order to check each scenario, PBl was implemented in seven steps (Table 1). At the beginning of each session, the scenario of the last week was represented and analyzed. At the end of the session, the next week's scenario was given to the students. One month after completion of the training sessions, the questionnaire of patient safety-based communication skills was recompleted by students in the two groups.

\section{Data analysis}

Data analysis was performed in SPSS version 16.0 (SPSS Inc., Chicago, IL) using descriptive (Percent, Frequency, Mean, and standard deviation) and inferential (chi-square and independent and paired $t$ test) statistics before and after the intervention. The consort flow diagram for this study is presented in Figure 1. 
Table 1. Implementation stages of PBL

\begin{tabular}{|c|c|}
\hline First Stage & Reading the scenario and encouraging students to clarify the ambiguities \\
\hline Second Stage & Elaboration on the subject by the researcher \\
\hline Third Stage & Brainstorming, group participation, and discussion about the subject \\
\hline Fourth Stage & $\begin{array}{l}\text { Listing the facts and hypothesizing based on the scenario contents and responding to the questions based on the nursing process to achieve } \\
\text { better educational goals }\end{array}$ \\
\hline Fifth Stage & Intragroup agreement on learning goals and the instructor's assurance of achieving complete, comprehensive, and appropriate goals \\
\hline Sixth Stage & Individual and group study to collect information from the sources introduced by the students in the library and through the internet \\
\hline Seventh Stage & $\begin{array}{l}\text { Presenting the determined subjects based on the hypotheses, goals, and questions; conducting group discussions; drawing conclusions and } \\
\text { assessing the presented subjects by the instructor }\end{array}$ \\
\hline
\end{tabular}

Results

The results of independent $t$ test and chi-square indicated no significant differences between the intervention and control groups in terms of age, gender, semester, marital status, place of residence, interest in the discipline, clinical work experience, and grade point average (Table 2).

The results demonstrated no significant difference between the intervention and control groups in terms of the mean scores of the patient safety-based communication skills before the intervention $(P=0.123)$, while a significant difference was observed in this regard between the groups after the intervention $(P=0.001$; Table 3$)$.

In the control groups, no significant difference was observed in the mean score of patient safety-based communication skills before and after the intervention $(P=0.162)$. On the other hand, the intervention groups had significantly different scores after the educational sessions $(P=0.001$; Table 4$)$.

\section{Discussion}

The results showed no significant difference between the intervention and control groups in terms of patient safetybased communication skills before the intervention. However, a significant difference was observed in the score of the patient safety-based communication skills of the students in the intervention groups after the PBL sessions. Therefore, it seems that the PBL method could enhance the patient safety-based communication skills of the nursing students, which is in line with the study by Mansouri et al, who claimed that the PBL approach positively influenced the educational performance of students compared to conventional education (20). In a review study, Haj Babaei and Ashrafizadeh stated that several studies had confirmed the efficacy of the PBL method in nursing education and student empowerment, demanding the expansion and comprehensive use of this approach in nursing education (21). Similar to our findings, Dring asserted that clinical

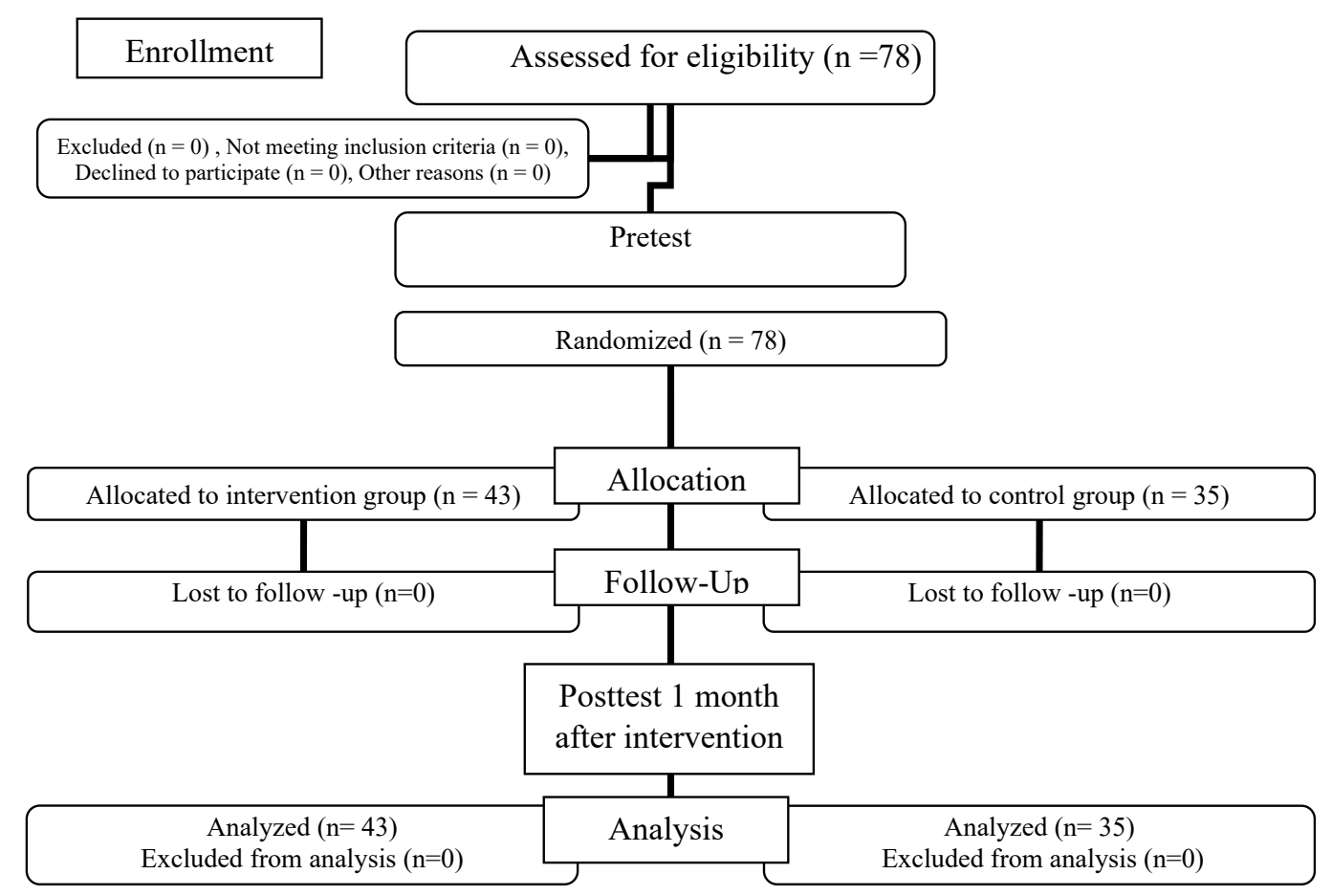

Figure 1. The consort flow diagram of study. 
Table 2. Comparison of intervention and control groups in terms of demographic characteristics

\begin{tabular}{|c|c|c|c|c|}
\hline Variable & & Control group & Intervention group & Test results \\
\hline \multirow{2}{*}{ Gender, No. $(\%)$} & Male & $12(33.3)$ & $24(66.7)$ & \multirow{2}{*}{$\begin{array}{c}\chi^{2}=3.59 \\
P=0.058^{\mathrm{a}}\end{array}$} \\
\hline & Female & $23(54.8)$ & $19(45.2)$ & \\
\hline \multirow{2}{*}{ Semester, No. (\%) } & Seven & $14(38.9)$ & $22(61.1)$ & \multirow{2}{*}{$\begin{array}{c}\chi^{2}=0.96 \\
P=0.325^{\text {a }}\end{array}$} \\
\hline & Eight & $21(50)$ & $21(50)$ & \\
\hline \multirow{2}{*}{ Marital status, No. (\%) } & Single & $32(47.1)$ & $36(52.9)$ & \multirow{2}{*}{$\begin{array}{c}\chi^{2}=1.02 \\
P=0.311^{\text {a }}\end{array}$} \\
\hline & Married & $3(30)$ & $7(70)$ & \\
\hline \multirow{2}{*}{ Place of residence, No. (\%) } & Dormitory & $23(45.1)$ & $28(54.9)$ & \multirow{2}{*}{$\begin{array}{l}\chi^{2}=0.003 \\
P=0.956^{\mathrm{a}}\end{array}$} \\
\hline & Non- dormitory & $12(44.4)$ & $15(55.6)$ & \\
\hline \multirow{2}{*}{$\begin{array}{l}\text { Interest in the discipline, No. } \\
(\%)\end{array}$} & Yes & $22(45.8)$ & $26(54.2)$ & \multirow{2}{*}{$\begin{array}{l}\chi^{2}=0.047 \\
P=0.849^{a}\end{array}$} \\
\hline & No & $13(43.3)$ & $17(56.7)$ & \\
\hline \multirow{2}{*}{$\begin{array}{l}\text { Clinical work experience, No. } \\
(\%)\end{array}$} & Yes & $3(60)$ & $2(40)$ & \multirow{2}{*}{$\begin{array}{l}\chi^{2}=0.494 \\
P=0.482^{\text {a }}\end{array}$} \\
\hline & No & $32(43.8)$ & $41(56.2)$ & \\
\hline \multicolumn{2}{|l|}{ Age (years), Mean $\pm S D$} & $23.26 \pm 1.40$ & $22.95 \pm 1.64$ & $\begin{aligned} \mathrm{t} & =0.765 \\
P & =0.390^{\mathrm{b}}\end{aligned}$ \\
\hline \multicolumn{2}{|c|}{ Grade point average (GPA), Mean \pm SD } & $16.01 \pm 0.77$ & $16.08 \pm 1.14$ & $\begin{aligned} \mathrm{t} & =0.364 \\
P & =0.717^{\mathrm{b}}\end{aligned}$ \\
\hline
\end{tabular}

${ }^{\text {a }}$ Chi-square test; ${ }^{\text {b }}$ Independent $t$ test.

Table 3. Comparison of mean scores of patient safety-based communication skills between the intervention and control groups before and after PBL Sessions

\begin{tabular}{|c|c|c|c|}
\hline \multirow{2}{*}{ Communication skills (16-80) } & Control group & Intervention group & \multirow{2}{*}{ Test results } \\
\hline & Mean \pm SD & Mean \pm SD & \\
\hline Before Intervention & $59.05 \pm 17.55$ & $63.74 \pm 8.16$ & $\mathrm{t}=1.55, P=0.123^{\mathrm{a}}$ \\
\hline After intervention & $55.60 \pm 9.44$ & $96.16 \pm 11.62$ & $\mathrm{t}=5.56, P=0.0001^{\mathrm{a}}$ \\
\hline
\end{tabular}

${ }^{\mathrm{a}}$ Independent $t$ test.

Table 4. Comparison of mean scores of patient safety-based communication skills within the intervention and control groups before and after PBL Sessions

\begin{tabular}{|c|c|c|c|}
\hline \multirow{2}{*}{ Communication skills (16-80) } & Before Intervention & After intervention & \multirow{2}{*}{ Test results } \\
\hline & Mean \pm SD & Mean \pm SD & \\
\hline Control group & $59.05 \pm 17.55$ & $55.60 \pm 9.44$ & $\mathrm{t}=1.42, P=0.162^{\mathrm{a}}$ \\
\hline Intervention group & $63.74 \pm 8.16$ & $96.16 \pm 11.62$ & $\mathrm{t}=3.44, P=0.0001^{\mathrm{a}}$ \\
\hline
\end{tabular}

a Paired $t$ test.

and PBL practices could prepare students for cooperation and effective communication to provide patient-oriented services. The researcher also stated that implementing the method from the beginning of academic education creates implicit clinical knowledge and judgment, but it also increases the self-confidence and independence of students (22). In another research, Komasawa et al (2018) detected that using the PBL method could improve selfbelief in anesthesia residents of the operating room in the management of emergencies (23). In addition, Kan $\mathrm{Ku}$ and Michael Ha emphasized the necessity of changing the teaching philosophy among educators in various fields, such as medicine, nursing, and engineering, because of the efficacy of the PBL method (24). Khatiban et al also conducted a research to evaluate the efficacy of lecture and PBL in teaching ethics to nursing students, suggesting that the PBL method should be applied in other nursing areas, considering its efficiency in developing moral reasoning in students (25). In a review study performed by $\mathrm{Li}$ et al, limited evidence was proposed that PBL could enhance the professional communication skills of nurses and nursing students more effectively than conventional teaching methods (26). The results obtained by Liu et al demonstrated that PBL was superior to conventional teaching methods in domains such as interest in learning, team spirit at work, problem-solving ability, analysis, range of knowledge, communications, and expression (27). Additionally, the results of a review study by Sayyah et al were indicative of the positive impact of PBL on the academic advancement of medical students, proposing that more attention be paid to the efficiency of educational methods by the professors and decisionmakers of medical education (28). In a study by Yadav et al, PBL was reported to be an intriguing educational technique to medical students, playing a pivotal role in expanding the knowledge and skills of learning, as well as the development of interpersonal, communication, and expression skills. In addition, the role of professors as the facilitators of proper activities during educational sessions was highlighted in the mentioned research (29). While several studies have confirmed the efficiency of PBL, the study was conducted by Panjehpour and Ataee 
demonstrated no significant difference between the mean score of the PBL and professor-oriented groups, which is inconsistent with our findings (30).

\section{Conclusion}

In today's world, problem-solving and decision-making skills are the required knowledge and skills for nursing and midwifery students. The participation of students in active educational methods (e.g., PBL) increases their self-confidence, thereby leading to profound learning. Since problem-solving skills are recognized at the highest human cognitive level and the most valuable educational goals, it is recommended that nursing professors apply PBL in some courses as an alternative to conventional teaching methods to improve the clinical and cognitive abilities of nursing students. Thus, nursing schools could implement educational courses to teach professors about such approaches and emphasize their importance to nursing students, who will be employed in clinical environments imminently.

\section{Limitations of the study}

In the present study, the follow-up period was one month, and the short period of the study was one of the limitations, therefore it is recommended to conduct a study with a more extended follow-up period through which the effect of $\mathrm{PBl}$ on the persistence of learning over time could be determined.

\section{Acknowledgments}

This study is derived from a master thesis in nursing (Registration no.9466). The authors would like to express their sincere appreciation to the research deputy of Urmia University of Medical Sciences for its support and all the participants for their cooperation in this study.

\section{Authors' contribution}

$\mathrm{HJ}$, NP and MHM were the principal investigators of the study. HJ and MHM participated in preparing the concept and design. NP and $\mathrm{HJ}$ revised the manuscript and critically evaluated the intellectual contents. All authors participated in preparing the final draft of the manuscript. All authors have read and approved the content of the manuscript and confirmed the accuracy or integrity of any part of the work.

\section{Conflicts of interest}

The authors declare that they have no conflict of interest.

\section{Ethical issues}

The research followed the tenets of the Declaration of Helsinki. The Ethics Committee of Urmia University of Medical Sciences approved this study. The institutional ethical committee at Urmia University of Medical Sciences approved all study protocols (IR. UMSU.REC.1398.219). Accordingly, written informed consent was taken from all participants before any intervention. This study was extracted from master's thesis in nursing of Hossein Jamshidi at this university (Thesis \#9466). Moreover, ethical issues (including plagiarism, data fabrication, double publication) have been completely observed by the authors.

\section{Funding/Support}

This study was financially supported by the research deputy of Urmia University of Medical Sciences (Grant \#9466).

References

1. Austin JM, D'Andrea G, Birkmeyer JD, Leape LL, Milstein A, Pronovost PJ, et al. Safety in numbers: the development of Leapfrog's composite patient safety score for U.S. hospitals. J Patient Saf. 2014;10:64-71. doi: doi: 10.1097/ PTS.0b013e3182952644

2. Rezaean M, Aqaie Borz Abad P, Yazdanpanah A, Zinat Motlagh S. Patient safety culture status from the perspective medical staff of yasuj hospitals in 2015. Armaghan J. 2016;20:935-46.

3. Khalilzadeh H, Zare Fazlollahi Z, Mohaddesi H, Hemmati Maslak Pak M. Attitude among urmia medical university health workers about patient safety. Nurs Midwifery J. 2013;11:60613.

4. Hassan M, Ahmed T. Patient safety assessing nurses' compliance. J Am Sci. 2012;8:748-55.

5. Vazin A, Delfani S. Medication errors in an internal intensive care unit of a large teaching hospital: a direct observation study. Acta Med Iran. 2012;50:425-32.

6. Brasaitè I, Kaunonen M, Martinkènas A, Mockienè V, Suominen T. Health care professionals' skills regarding patient safety. Medicina (Kaunas). 2016;52:250-256. doi: 10.1016/j. medici.2016.05.004.

7. Arora S, Sevdalis N, Ahmed M, Wong H, Moorthy K, Vincent C. Safety skills training for surgeons: A half-day intervention improves knowledge, attitudes and awareness of patient safety. J Surg. 2012;152:26-31. doi: 10.1016/j.surg.2012.02.006.

8. White N. Understanding the role of non-technical skills in patient safety. Nurs Stand. 2012;26:43-8. doi: 10.7748/ ns2012.02.26.26.43.c8972.

9. Gordon M, Darbyshire D, Baker P. Non-technical skills training to enhance patient safety: a systematic review. Med Educ. 2012;46:1042-54. doi: 10.1111/j.1365-2923.2012.04343.x.

10. Odell M. Human factors and patient safety: changing roles in critical care. Aust Crit Care. 2011;24:215-7. doi: 10.1016/j. aucc.2011.02.001.

11. Brasaite I, Kaunonen M, Suominen T. Healthcare professionals' knowledge, attitudes and skills regarding patient safety: a systematic literature review. Scand J Caring Sci. 2015;29:3050. doi: $10.1111 / \mathrm{scs} .12136$

12. Leung GK, Patil NG. Patient safety in the undergraduate curriculum: medical students' perception. Hong Kong Med J. 2010;16:101-5.

13. O'Leary D, Leape L. Teaching physicians to provide safe patient care. Patient Safety Handbook. 2013:425.

14. Usher K, Woods C, Parmenter G, Hutchinson M, Mannix J, Power $\mathrm{T}$, et al. Self-reported confidence in patient safety knowledge among Australian undergraduate nursing students: A multi-site cross-sectional survey study. Int J Nurs Stud. 2017;71:89-96. doi: 10.1016/j.ijnurstu.2017.03.006.

15. Srinivasan M, Wilkes M, Stevenson F, Nguyen T, Slavin S. Comparing problem-based learning with case-based learning: effects of a major curricular shift at two institutions. Acad Med. 2007;82:74-82. doi: 10.1097/01.ACM.0000249963.93776. aa.

16. Mete S, Yildirim Sari H. Nursing students' expectations from tutors in PBL and effects of tutors' behaviour on nursing students. Nurse Educ Today. 2008;28:434-42. doi: 10.1016/j. nedt.2007.07.008.

17. Hemmati Maslak Pak M, Orujlu S, Khalkhali H. The effect of problem-based learning training on nursing students' critical thinking skills. J Med Educ. 2014;9:24-33. 
18. Nabilou B, Feizi A, Seyedin H. Patient Safety in Medical Education: Students' Perceptions, Knowledge and Attitudes. PLoS One. 2015;10:e0135610. doi: 10.1371/journal. pone. 0135610 .

19. Hemmati M, Sheikhbaglu M, Baghaie R. Relationship between the communication skill of nurse - patient with patient safety in the critical care units. J Clin Nurs Midwifery. 2014;3:77-84.

20. Mansoori S, Abedini-baltork M, Lashkari H, Bagheri S. Effectiveness of problem-based learning on student's academic performance: A quasi-experimental study. Res Med Educ. 2017;9:8-1. doi: 10.18869/acadpub.rme.9.1.8

21. Hajibabaee F, Ashrafizadeh H. A comprehensive review of problem-based learning in the Iranian nursing education. Iran J Nurs Res. 2019;32:11-28. doi: 10.29252/ijn.32.118.11

22. Dring JC. Problem-Based Learning - Experiencing and understanding the prominence during Medical School: Perspective. Ann Med Surg (Lond). 2019;47:27-8. doi: 10.1016/j.amsu.2019.09.004.

23. Komasawa N, Berg BW, Minami T. Problem-based learning for anesthesia resident operating room crisis management training. PLoS One. 2018;13:e0207594. doi: 0.1371/journal. pone.0207594

24. Ku TK, Ha M. The application of problem based learning in undergraduate nursing education: A strategy for curriculum reform. J Biosci Med. 2016;4:52-9. doi: 10.4236/ jbm.2019.71004.

25. Khatiban M, Falahan SN, Amini R, Farahanchi A, Soltanian
A. Lecture-based versus problem-based learning in ethics education among nursing students. Nurs Ethics. 2019;26:175364. doi: 10.1177/0969733018767246.

26. Li $Y$, Wang $X$, Zhu $X R$, Zhu YX, Sun J. Effectiveness of problem-based learning on the professional communication competencies of nursing students and nurses: A systematic review. Nurse Educ Pract. 2019;37:45-55. doi: 10.1016/j. nepr.2019.04.015.

27. Liu L, Du X, Zhang Z, Zhou J. Effect of problem-based learning in pharmacology education: A meta-analysis. Studies in Educational Evaluation. Br J Educ Stud. 2019;60:43-58. doi. org/10.1016/j.stueduc.2019.100813.

28. Sayyah M, Shirbandi K, Saki-Malehi A, Rahim F. Use of a problem-based learning teaching model for undergraduate medical and nursing education: a systematic review and metaanalysis. Adv Med Educ Pract. 2017;8:691-700. doi: 10.2147/ amep.s143694.

29. Yadav RL, Piryani RM, Deo GP, Shah DK, Yadav LK, Islam MN. Attitude and perception of undergraduate medical students toward the problem-based learning in Chitwan Medical College, Nepal. Adv Med Educ Pract. 2018;9:317-22. doi: 10.2147/amep.s160814

30. Panjehpour M, Ataee N. Comparison the effectiveness of problem solving method with lecture-based method in the teaching of metabolic biochemistry. Iran J Med Educ. 2012;11:1318-25. 